\title{
Melan-A Expression in Poorly Differentiated Carcinoma of Lung: A Potential Diagnostic Pitfall
}

wenyong huang ( $\nabla$ wenyongh2009@yeah.net)

Nanchang University Second Affiliated Hospital

\section{Shuixian Li}

Jiujiang Health school

\section{Guofeng Zhu}

Nanchang University Second Affiliated Hospital

\section{Lei Zeng}

Nanchang University Second Affiliated Hospital

\section{Yueer Zheng}

Nanchang University Second Affiliated Hospital

Tao Huang

Nanchang University Second Affiliated Hospital

\section{Wen Zhang}

Nanchang University Second Affiliated Hospital

\section{Shousheng Liao}

Nanchang University Second Affiliated Hospital

Fanrong Liu

Nanchang University Second Affiliated Hospital

\section{Lixiang Li}

Nanchang University Second Affiliated Hospital

\section{Case Report}

Keywords: Melan-A, poorly differentiated carcinoma, lung

Posted Date: September 14th, 2021

DOl: https://doi.org/10.21203/rs.3.rs-864613/v1

License: (9) This work is licensed under a Creative Commons Attribution 4.0 International License. Read Full License 


\section{Abstract}

\section{Background}

Melan-A/MART-1 is a melanocytic differentiation marker, which is recognized as an antigen on melanoma cells. It is relevant for pathologists as a useful diagnostic marker in the diagnosis of melanocytic tumors. However, the expressional pattern of Melan-A in poorly differentiated carcinoma of lung has never been reported so far.

Case presentation

Here, we report a 77-year-old female patient who presented with a large mass in the right lung and was subsequently diagnosed with a poorly differentiated carcinoma of lung. We unexpectedly found that the carcinoma in this patient exhibited diffuse Melan-A expression.

\section{Conclusion}

This is the first case reported of a poorly differentiated carcinoma of lung with Melan-A expression. This report shows that Melan-A can express in poorly differentiated carcinoma, and highlights a potential diagnostic pitfall in the diagnosis of carcinoma, which urges pathologists to exercise caution in cases where Melan-A positivity and illustrates the need for further immunohistochemical or molecular examination to avoid misdiagnosis.

\section{Background}

The Melan-A gene, cloned from human melanoma cell line SK-MEL-29, encodes a melanoma antigen recognized by autologous cytotoxic T cells [1]. By analyzing the antigenic targets of tumor-infiltrating lymphocytes from a melanoma specimen, independently cloned the same gene, designated MART-1 [2]. The Melan-A protein is a melanocytic differentiation antigen, product of the MART-1 gene, and is thought to be specific for melanocytic cells [3]. It was found to be a useful addition to antibody panels for melanocytic neoplasms and it is of interest for clinicians as potential immunotherapeutic target [4].

Except for positive expression of melanocytic neoplasms, the Melan-A antigen can also express in adrenal tissue, sex cord stromal tumours and MiT family translocation renal cell carcinoma $[5,6]$. Besides, the expression of Melan-A in breast carcinoma has been reported recently. In their study, immunohistochemically focally positive cells for the melanocytic marker Melan-A showed in 18 out of 100 breast carcinomas. Microarrays of a further 159 breast cancers showed, in several samples, variably low expression levels of Melan A [7].

Melanoma markers expressed in non-small cell lung cancer (NSCLC) has also been reported in a recent research of English literature, the results reveal HMB45, MelanA, and SOX10 were positive in $1(<1 \%), 0$ $(0 \%)$ and $5(<1 \%)$ cases in 1027 NSCLC tumor tissue samples [8]. Thus, the differential diagnosis between melanoma and poorly differentiated carcinoma could be confusing as their similar morphology, 
especially when the melanoma markers expressed in both of them. Although several melanoma markers have been reported to be expressed in NSCLC, the expression of Melan-A in lung cancer has not been reported so far.

Herein we reported an extremely rare case of poorly differentiated carcinoma of lung, diffuse positivity for Melan-A. Our case highlights a potential diagnostic pitfall in the diagnosis of poorly differentiated carcinoma.

\section{Case Presentation}

A 77-year-old female patient presenting with cough for more than 10 days, chest pain for 2 days was admitted to our hospital. The patient had a history of hepatitis B cirrhosis and long-term use of entecavir. She had no history of melanoma. Computed tomography (CT) showed irregular soft tissue mass beside the right hilum. The cross-section size of the lung window was about $41 * 28 \mathrm{~mm}$, the lesion was lobulated and significantly enhanced on the enhanced scan. Multiple enlarged lymph nodes were seen in the right supravian fossa and mediastinum, but there was no obvious mass was found on the rest of her body. A bronchoscopic puncture biopsy was performed to determine the nature of the tumor, which located in the right intermediate bronchus. Histologically, epithelioid tumor cells were arranged in a prominent nesting, cords, or glandular tubules pattern (Fig. 1a and 1b). Tumor cells were positive for pancytokeratin (Fig. 1C), CK7 (Fig. 1d), CK19, EMA (Fig. 1e), Melan-A (Fig. 1f), and CK20 (focal positive), but negative for TTF-1, Napsin-A, P40, P63, S-100 (Fig. 2a), HMB-45 (Fig. 2b), CD117 (Fig. 2c), SMA, a-inhibin, CK5/6, D2-40, Calretinin, SALL-4, OCT-4, CD30, CgA, Syn, CD56, ALK-D5F3 and CDX-2. Ki-67 labeling was estimated to be $60 \%$ (Fig. 2d). These findings, especially the presence of pancytokeratin, CK7, CK19 and EMA, supported the diagnosis of poorly differentiated carcinoma. To exclude the possibility of metastatic malignant melanoma, the melanoma markers, S100 and HMB45, were used in this case and all of them were negative. What's more, the absence of a history of melanoma does not support the diagnosis of metastatic malignant melanoma. In addition, we performed genetic tests on this patient. 26 genes (EGFR, ALK, ROS1, BRAF, NRAS, MAP2K1, KRAS, PIK3CA, AKT1, CTNNB1, ERBB2, ERBB4, FGFR1, FGFR3, FGFR2, SMAD4, MET, NOTCH1, NTRK1, DDR2, PTEN, RET, STK11, TP53, FBXW7, UGT1A1) were identified by high-throughput sequencing technology (Semiconductor sequencing). Our results showed no mutation of BRAF, NRAS and MAP2K1 in this patient, which are closely associated with melanoma $[9,10]$. Interestingly, we found that the patient just revealed presence of p.Y205C mutation in exon 6 of TP53. Finally, the patient did not receive any chemotherapy, and died two months later.

\section{Discussion}

Melan-A protein is a melanocytic differentiation antigen, and is thought to be specific for melanocytic cells. Besides, Melan-A can also express in several kinds of carcinomas such as MiT family translocation renal cell carcinoma and breast cancers [6, 7]. However, there was no data available describing the expressional pattern of Melan-A in lung cancer. Here, we present a case of poorly differentiated carcinoma expressed Melan-A that occurred in the right lung. Pathological examination revealed that the 
tumor mainly consisted of epithelioid cells arranged in a prominent nesting, cords, or glandular tubules pattern. Based on the clinical history, morphology and immunohistochemical staining of the tumor cells, we excluded the possibility of metastatic melanoma and finally made a diagnosis of poorly differentiated carcinoma.

In fact, the differential diagnoses between poorly differentiated carcinoma and malignant melanoma are challenging sometimes. They have similar morphologic characteristics include epithelioid tumor cells arranged in a prominent nesting or cords pattern, especially for the small biopsy specimens. At this time, we need to use clinical informations, more extensive immunohistochemical pannel, and even molecular detection methods to assist diagnosis. The present patient showed glandular growth pattern at least focally, positive for pancytokeratin, CK7 and EMA expression but negative for S100, HMB45, and the molecular detection revealed no mutation of BRAF, NRAS and MAP2K1 $[9,10]$, all these above are very helpful for us to make a relatively reasonable diagnosis.

Given the overlapping immunohistochemical expression between carcinoma and melanoma, diagnosis of poorly differentiated carcinoma or malignant melanoma should be made with great caution and warrants exploration of the remote history. Genotyping is a helpful surrogate marker in classifying such difficult cases. According to a recent report in literature, metastatic melanomas can show complete loss of immunohistochemical melanocytic markers such as S100, HMB45, Melan A, and SOX10, and can even abnormally express pancytokeratin [11,12], which urges pathologists to exercise caution in cases of poorly differentiated carcinoma and illustrates the need for further immunohistochemical or molecular examination to avoid misdiagnosis.

In summary, we reported a rare case of poorly differentiated carcinoma of lung with Melan-A expression, which highlights a potential diagnostic pitfall in our daily work. The mechanism underlying Melan-A expression in these tumors is still unclear, further studies are warranted to elucidate the mechanism and clinical significance of Melan-A expression.

\section{Abbreviations}

NSCLC: non-small cell lung cancer;

CT: Computed tomography.

\section{Declarations}

\section{Acknowledgements}

Not applicable.

\section{Conflict of interest}

The authors disclose no conflicts. 


\section{Funding sources}

No funding or support is associated with this study.

\section{Authors' contribution}

Wenyong Huang contributed in collecting clinical data and writing the paper. Shuixian Li provided H\&E staining images. Guofeng Zhu provided imaging and clinical information. Shousheng Liao and Wen Zhang performed the immunohistochemistry staining and molecular tests experiments. Lei Zeng, Yueer Zheng, Tao Huang, Fanrong Liu and Lixiang Li designed the study. All authors read and approved the final manuscript.

\section{Availability of data and materials}

The data and materials are available upon request.

\section{Ethics approval and consent to participate}

This publication is approved by the Ethics Committee of the Second Affiliated Hospital of Nanchang University.

\section{Consent for publication}

Consent from the patient is obtained.

\section{Author details}

${ }^{1}$ Department of Pathology, The Second Affiliated Hospital of Nanchang University, No.1, Minde Road, Donghu District, Nanchang 330000, China.

${ }^{2}$ Office of Teaching Discipline, Jiujiang Health School, No.1272, Shili Avenue, Lianxi District, Jiujiang 332000 , China.

\section{References}

1. Coulie PG, Brichard V, Van Pel A, et al.A new gene coding for a differentiation antigen recognized by autologous cytolytic T lymphocytes on HLA-A2 melanomas. J Exp Med. 1994; 180(1):35-42.

2. Y Kawakami, S Eliyahu, $\mathrm{CH}$ Delgado, et al. Cloning of the gene coding for a shared human melanoma antigen recognized by autologous T cells infiltrating into tumor. Proc Natl Acad Sci U S A. 1994; 91(9):3515-9.

3. Chen YT, Stockert E, Jungbluth A, et al. Serological analysis of Melan-A(MART-1), a melanocyte-specific protein homogeneously expressed in human melanomas. Proc Natl Acad Sci U S A. 1996; 93:59155919. 
4. Blessing K, Sanders DS, Grant JJ. Comparison of immunohistochemical staining of the novel antibody melan-A with S100 protein and HMB-45 in malignant melanoma and melanoma variants. Histopathology. 1998; 32:139-146.

5. C J R Stewart, C L Nandini, J A Richmond. Value of A103 (melan-A) immunostaining in the diVerential diagnosis of ovarian sex cord stromal tumours. J Clin Pathol. 2000; 53(3): 206-211.

6. Pedram Argani. MiT family translocation renal cell carcinoma. Int J Oncol. 2008; 33(5):1011-5.

7. Beatrice E Bachmeier, Andreas G Nerlich, Valentina Mirisola, et al. Lineage infidelity and expression of melanocytic markers in human breast cancer. Int J Oncol. 2008;33(5):1011-5.

8. Mark Kriegsmann, Katharina Kriegsmann, Alexander Harms, et al. Expression of HMB45, MelanA and SOX10 is rare in non-small cell lung cancer. Diagnostic Pathology, 2018; 13:68.

9. Erik A. Williams, Meagan Montesion, Nikunj Shah, et al. Melanoma with in-frame deletion of MAP2K1: a distinct molecular subtype of cutaneous melanoma mutually exclusive from BRAF, NRAS, and NF1 mutations. Modern Pathology, 2020; 33:2397-2406.

10. Hugo Akabane, Ryan J Sullivan. The Future of Molecular Analysis in Melanoma: Diagnostics to Direct Molecularly Targeted Therapy. Am J Clin Dermatol. 2016; 17(1):1-10.

11. Abbas Agaimy, Katja Specht, Robert Stoehr, et al. Metastatic Malignant Melanoma With Complete Loss of Differentiation Markers (Undifferentiated/Dedifferentiated Melanoma): Analysis of 14 Patients Emphasizing Phenotypic Plasticity and the Value of Molecular Testing as Surrogate Diagnostic Marker. Am J Surg Pathol. 2016; 40(2):181-91.

12. Abbas Agaimy, Robert Stoehr, Annkathrin Hornung, et al. Dedifferentiated and Undifferentiated Melanomas: Report of 35 New Cases with Literature Review and Proposal of Diagnostic Criteria. Am J Surg Pathol. 2021; 45(2):240-254.

\section{Figures}



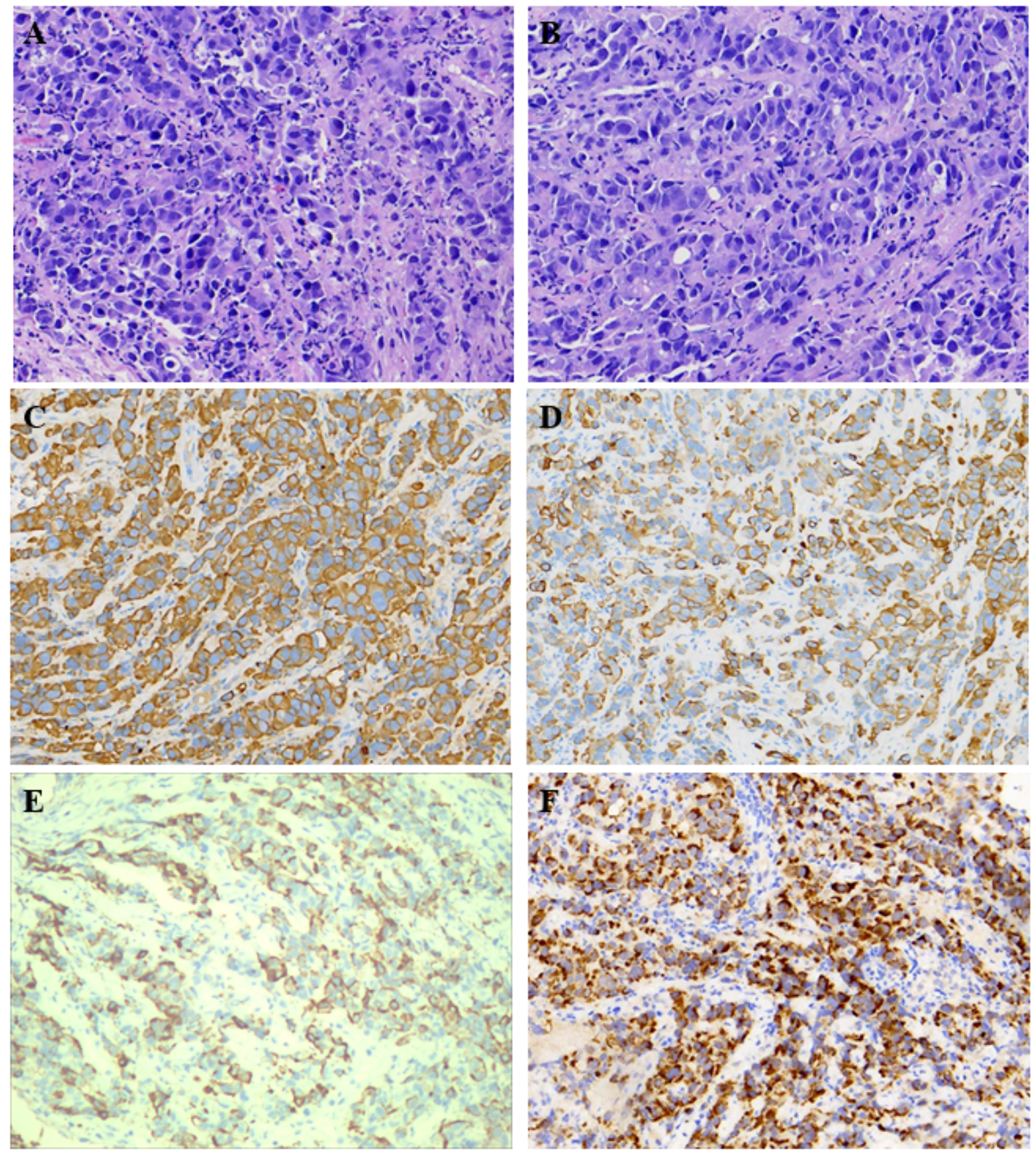

\section{Figure 1}

Morphological and immunohistochemical features of this poorly differentiated carcinoma. Histologically, the epithelioid tumor cells arranged in a prominent nesting (a) or glandular pattern (b). The tumor cells exhibited a diffuse strong positive expression for pancytokeratin (c), CK7 (d), EMA (e), and Melan-A (f). 

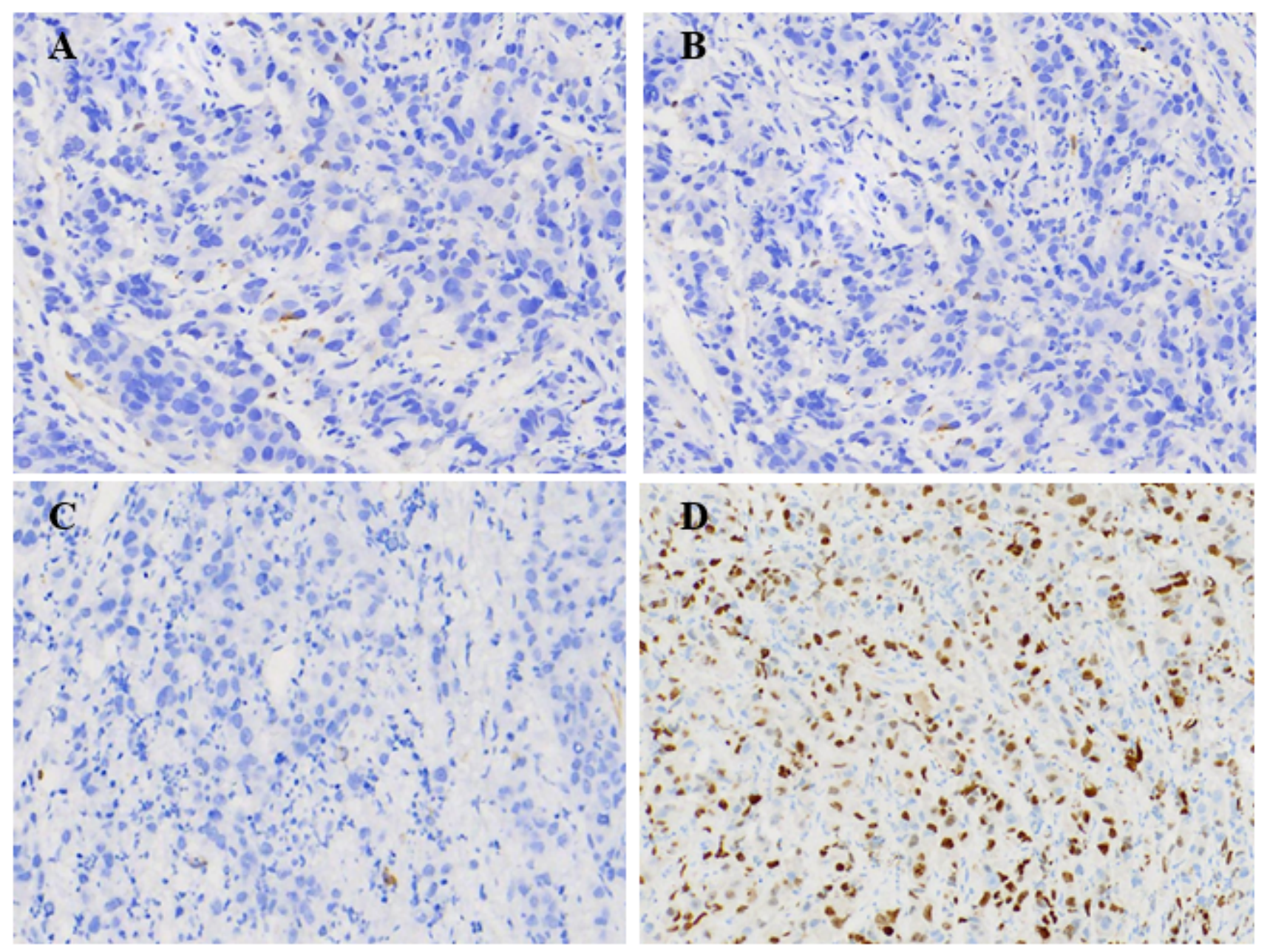

\section{Figure 2}

immunohistochemical features of this poorly differentiated carcinoma. S-100 (a), HMB45 (b) and CD117 (c) were negative expression in this patient, the Ki-67 labeling index was estimated to be $60 \%$ (d).

\section{Supplementary Files}

This is a list of supplementary files associated with this preprint. Click to download.

- CAREchecklist.doc 